\title{
Implementing the LFM-CW MIT Radar at the Ecuadorian Space Institute: Some Results
}

\author{
Alfonso Zozaya ${ }^{1, *}$, Rosa Bolaños²
}

Zozaya A iD https: / / orcid.org/0000-0003-3357-3887

How to cite

Zozaya A; Bolaños R (2020) Implementing the LFM-CW MIT Radar at the Ecuadorian Space Institute: Some Results J Aerosp Tecnol Manag, 12: e0520. https://doi.org/10.5028/jatm. v12.1091

ABSTRACT: This paper deals with the implementation of a version of the MIT LFM-CW radar at the Ecuadorian Space Institute. The effects of near and very near ranges, and free space loss in the performance of the image processing is theoretically revised. Subsequently, the implemented radar is tested in an imaging campaign on a small probing polygon, and very congruent results are obtained.

KEYWORDS: Instituto Espacial Ecuatoriano, Synthetic aperture radar, $\omega$-א algorithm.

\section{INTRODUCTION}

The Ecuadorian Space Institute, IEE (from its Spanish name Instituto Espacial Ecuatoriano), is an Ecuadorian institution, founded in 2012, considered the leader in research and development of the aerospace technology field in Ecuador. A roadmap for designing and implementing an operative radar for remote sensing purposes was delineated at IEE in 2017. This program is a long-term effort set for the time necessary to master research and development activities in the field of synthetic aperture radars (SAR).

As a first step in this journey, we decided to implement the MIT small linear frequency modulated (LFM) continuous wave (CW) SAR, very well documented in Fenn (2011), Williams (2011), and Charvat (2011a, 2011b, 2011c, 2011d, 2011e, 2011f, 2014). To meet this initial goal, the valuable experience of Brigham Young University (BYU) in designing small LFM-CW SAR was taken into account as well (Duersch 2004, Tolman 2008, Edwards 2009, Zaugg 2010).

Similar initiatives in other South American countries, to the best of our knowledge, have not been reported yet in the literature (Moreira et al. 2013), excepting the notorious Satélite Argentino de Observación Con Microondas (SAOCOM), which houses a fully operational L-band polarimetric synthetic aperture radar. The Argentinian Space Agency (CONAE) decided to build and operate a SAR Mission in 1996. From then to 1999, several meetings with potential users from different fields, such as emergencies, agriculture, ecology, forestry, glaciology, geology, oceanography, etc., were organized by CONAE, and the general specifications of the radar were established (Giraldez 2004). The SAOCOM 1a, the first of two identical satellites, was launched finally in October $7^{\text {th }}, 2018$.

\footnotetext{
三 1. Universidad Tecnológica Metropolitana - Departamento de Electricidad - Santiago, Chile.

2. Instituto Espacial Ecuatoriano - Quito, Ecuador.

*Correspondence author: a.zozayas@utem.cl

Received: Dec. 18, 2018 | Accepted: Mar. 30, 2019

Section Editor: Alison Moraes
} 


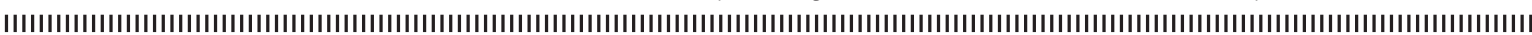

This paper deals with the implementation of a version of the LFM-CW radar developed at the MIT short radar course for both IAP (Independent Activities Period) and professional education (available in https://ocw.mit.edu/resources/).

The paper is structured as follows: in the second section a brief description of the MIT LFM-CW radar is given. The third section makes some considerations on focusing strategies. Near and very near ranges are also defined in this section, for the purpose of the analysis, and the performance of image processing is envisaged. The free space loss dynamic range is revised, and the range-migration/ $\omega-\kappa$ algorithm is briefly outlined. In the fourth section, some results are presented. Conclusions are given in the last section.

\section{MIT'S LFM-CW RADAR DESCRIPTION}

A detailed description of the MIT LFM-CW radar can be found in Charvat (2014); for completeness, a brief description is given as follows. Figures 1 and 2 show a simplified block diagram, and a snapshot of the radar implemented at IEE, respectively.

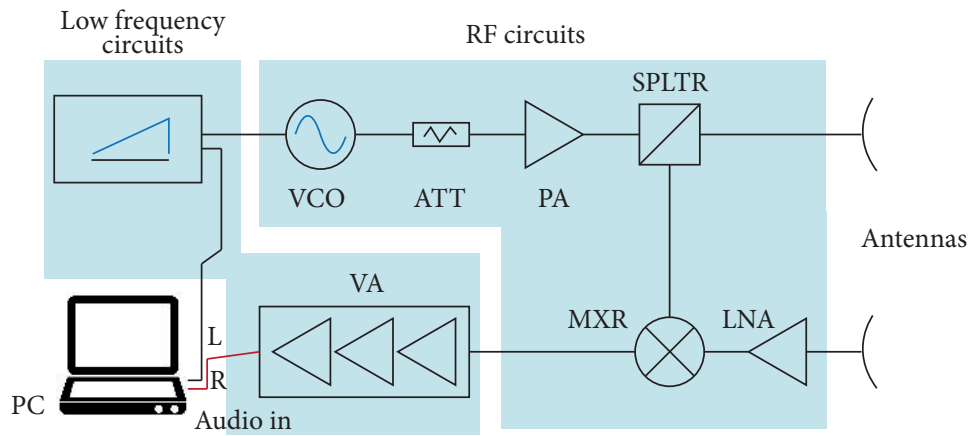

Figure 1. Block diagram.

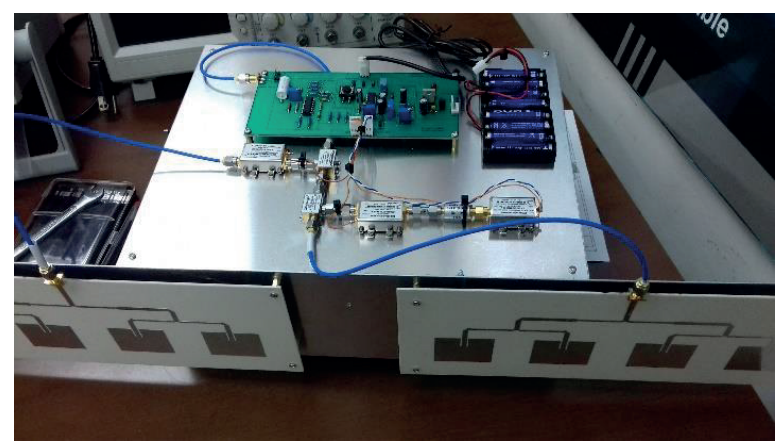

Figure 2. Snapshot of the radar implemented at IEE.

As it can be seen on Fig. 1, the radar has four major parts: a pair of antennas, the low and radio frequency circuits, and a personal computer (PC). A triangular signal is generated on the low frequency circuits to feed the voltage-controlled oscillator (VCO) in order to produce an LFM signal with central frequency $f_{c}=2.4 \mathrm{GHz}$ and bandwidth BW $=24 \mathrm{MHz}$. After amplification in the power amplifier (PA) and bifurcation in the splitter (SPLTR), a signal $x(t)$ is radiated to free space by the Tx-antenna:

$$
x(t)=A \cos \left(2 \pi f_{0}+K \pi t^{2}\right)
$$


where $f_{0}=f_{c}-B W / 2$ is the initial frequency; and $K=24 \times 10^{9} \mathrm{~Hz} / \mathrm{s}$ is the chirp rate.

For a point target located at $\left(u_{0}, r_{0}\right)$ on the slant plane, as shown in Fig. 1, an echo $e(t)$ is received by the $\mathrm{Rx}$-antenna and amplified by the low-noise amplifier (LNA):

$$
e(t)=\alpha A \cos \left[2 \pi f_{0}(t-\Delta)+K \pi(t-\Delta)^{2}\right]+n(t)
$$

where $\Delta=2 r / c$ is the effective time of flight; $c$ is the speed of light $\left(c=3 \times 10^{8} \mathrm{~m} / \mathrm{s}\right)$; and $\sqrt{r_{0}^{2}+\left(u-u_{0}\right)^{2}}$ is the target effective distance, where $u=u(t)$ is the radar location (see Fig. 3), and $n(t)$ is white noise.

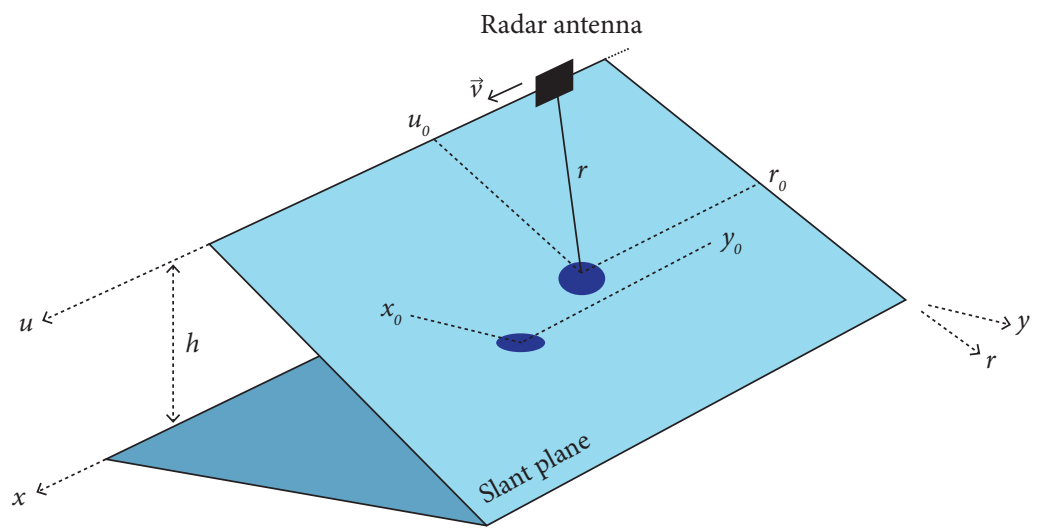

Figure 3. Point target and slant plane geometry.

After beating the echo $e(t)$ with a sample of the transmitted signal $x(t)$ in the mixer (MXR) and band-pass filtering the derived mixed signal in the video amplifier (VA), the dechirped signal $s(t)$ is obtained and captured in the PC via the audio input. Afterwards, the analytic signal $s_{\mathrm{A}}(t)$ is constructed in the PC by combining $s(t)$ with its Hilbert transform:

$$
S_{A}(t)=C e^{-j 2 \pi(K \Delta) t} e^{-j 2 \pi f_{0} \Delta} e^{-J R V P}
$$

where $K \Delta$ is the beating frequency of the target; $2 \pi f_{0} \Delta$ composes its azimuthal phase history; and $R V P$ is a residual video phase that can be neglected.

The samples of $s_{\mathrm{A}}(t)$ make up the raw data and feed the focusing strategies.

\section{FOCUSING CONSIDERATIONS AND STRATEGIES}

\section{NEAR AND VERY NEAR RANGES ANALYSIS}

In order to study the impact of a short radar trajectory length in the focusing process, we have defined two areas of interest: the near region (NR) area was defined as the set of distances from the radar between 100 and $750 \mathrm{~m}$; and the very near region (VNR), as the assembly of distances between 1 and $100 \mathrm{~m}$. Figure 4 shows the geometry of such a hypothetical probing region (VNR + NR) with two point targets. 


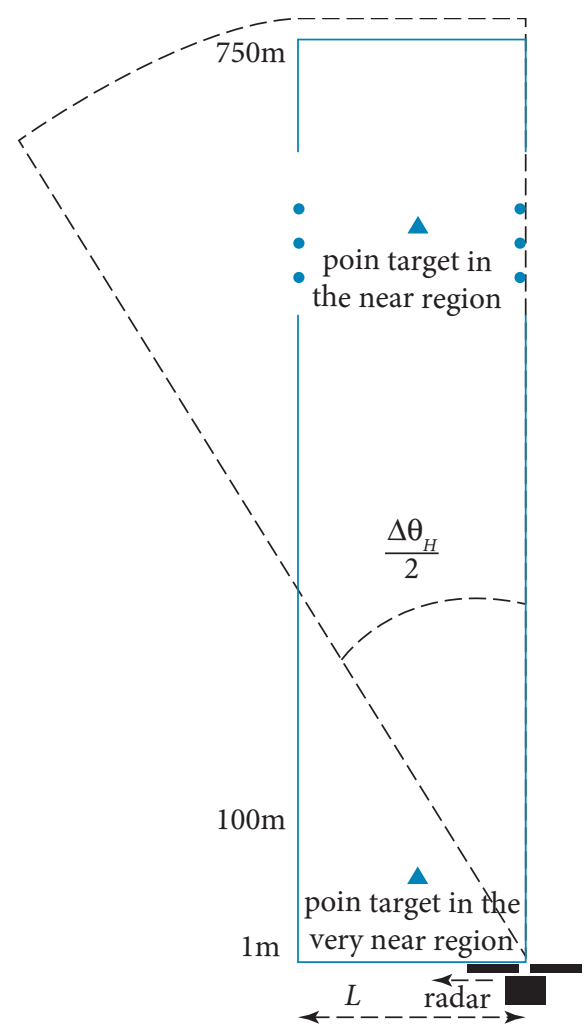

Figure 4. Probing polygon geometry.

As it is well known, the horizontal beam-width of the antenna and the distance traveled by the radar, in terms of the horizontal length of the antenna footprint, determines the richness of the azimuthal phase history registered for a given target. Likewise, the horizontal phase history defines the Doppler frequency support bandwidth for that target. If the radar trajectory length is lesser than the horizontal length of the antenna footprint, the quality of the azimuthal focusing procedure will depend greatly on the range of the target.

Let $L$ be the radar trajectory length. For targets located in any region, for a given horizontal beam-width $\Delta \theta_{\mathrm{H}}$, the smaller $L$ is, the poorer the phase history registered by the radar. In Fig. 5, the geometry of the region to be imaged for a smaller length trajectory $\left(L^{\prime}\right)$ is shown (Case A). In this case, the azimuthal history of a point target at the NR is shorter than that of a point target at the VNR.

On the other hand, for targets in any region, for a given small value of $L$, the bigger the azimuthal beam-width is, the poorer becomes the phase history registered for that target. Figure 6 depicts the geometric effect of incrementing the beam-width (Case B). The azimuthal history of both point targets is now shorter than the corresponding one in Case A.

Finally, for given values of (small) $L$ and $\Delta \theta_{\mathrm{H}}$, the closer to the radar (in the range-direction) and nearer to the $L$ midpoint (in the azimuth direction) the target is, the richer its phase registry becomes.

Figures 7 and 8 show the azimuthal phase histories $2 \pi f_{0} \Delta$ for several point targets situated at VNR and NR, respectively, all laid along the central line perpendicular to $L$. In these figures, $u(-5 m<u<5 m)$ is the radar coordinate defined over its trajectory axis, so they correspond to a radar trajectory length of 10 meters $(L=100 \mathrm{~m})$. 


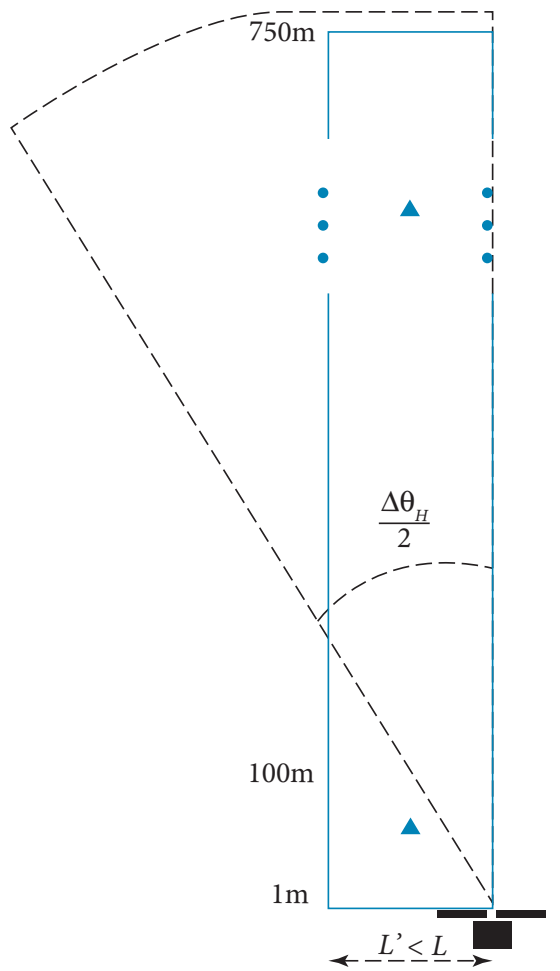

Figure 5. Case $A: L^{\prime}<L$

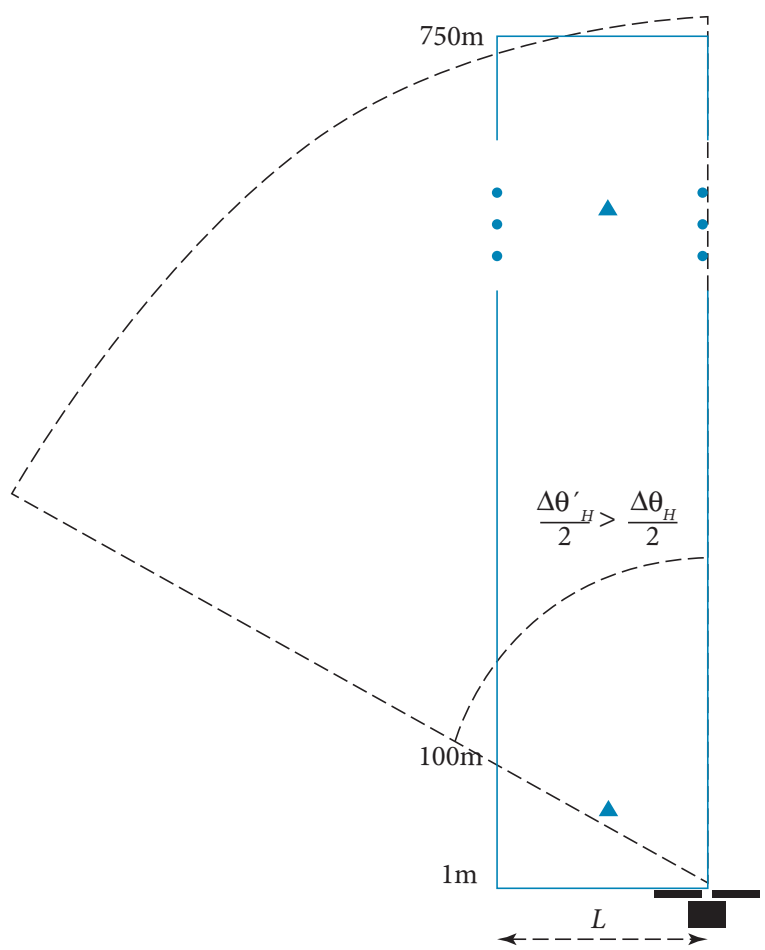

Figure 6. Case $\mathrm{B}: \frac{\Delta \theta_{H}^{\prime}}{2}>\frac{\Delta \theta_{H}}{2}$. 


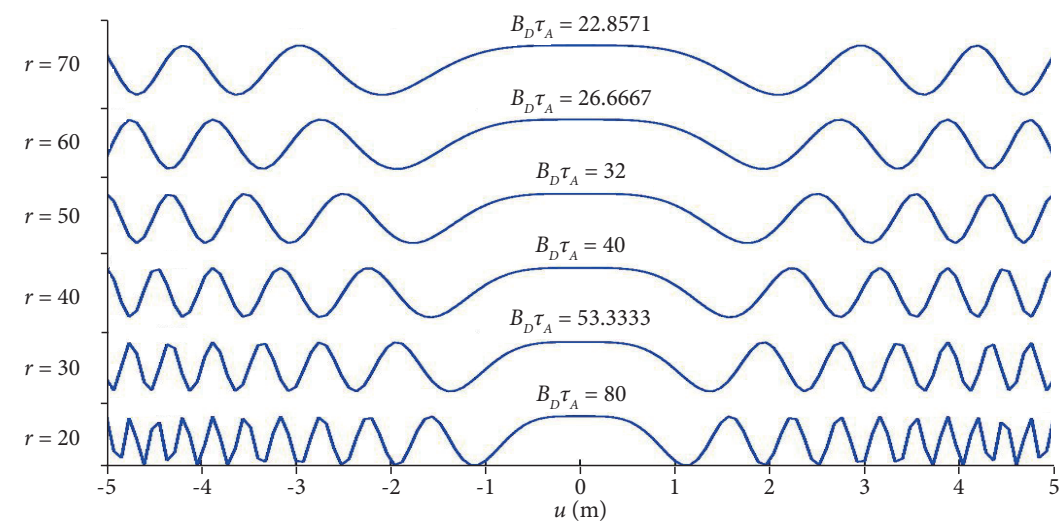

Figure 7. Azimuthal phase histories $\cos \left(2 \pi f_{0} \Delta\right)$ for several point targets at the VNR.

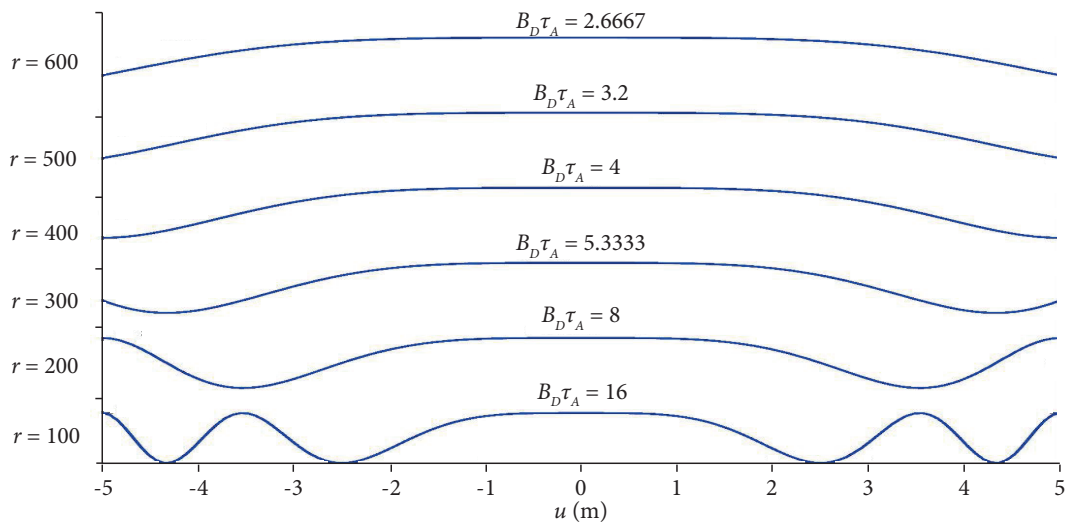

Figure 8. Azimuthal phase histories $\cos 2 \pi f_{0} \Delta$ for several point targets at the NR.

For targets at the NR, the Doppler bandwidth $\left(B_{\mathrm{D}}\right)$ and illumination time $\left(\tau_{\mathrm{A}}\right)$, the product $\left(B_{\mathrm{D}} \tau_{\mathrm{A}}\right)$ is very small compared to 100. For this reason, any focusing strategy will either fail or produce a very poor compression. Figure 9 displays the resulting sinclike functions from azimuth-matched filtering.

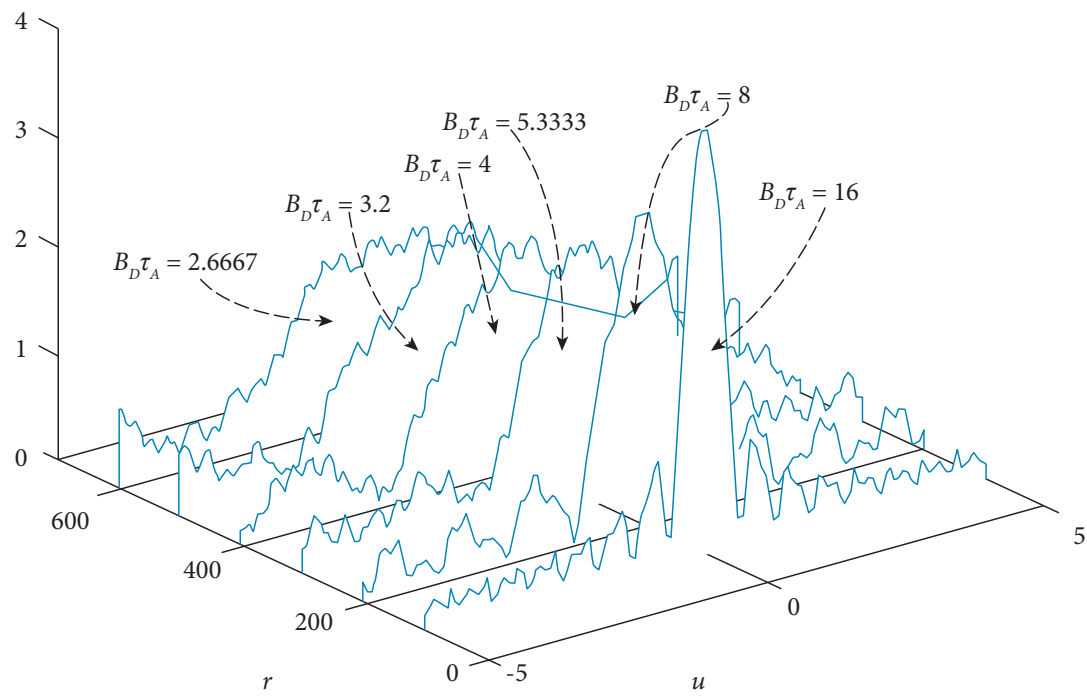

Figure 9. Sinc-like functions resulting from azimuth-matched filtering for targets located at the NR. 
As can be seen on Fig. 9, the nearest target at the NR is better focused than the others because it exhibits a greater $B_{\mathrm{D}} \tau_{\mathrm{A}}$. The focusing of the rest of the targets becomes worse as their range increases and their $B_{\mathrm{D}} \tau_{\mathrm{A}}$ diminishes.

Figure 10 shows the sinc-like functions obtained from the azimuth-matched filtering procedure for targets at the VNR. In this case, the product $B_{\mathrm{D}} \tau_{\mathrm{A}}$ becomes larger than those for targets at the NR, but it could still be smaller than 100. However, the sinc-like functions obtained from azimuth-matched filtering are better defined, and the focusing procedure could be assumed as successful.

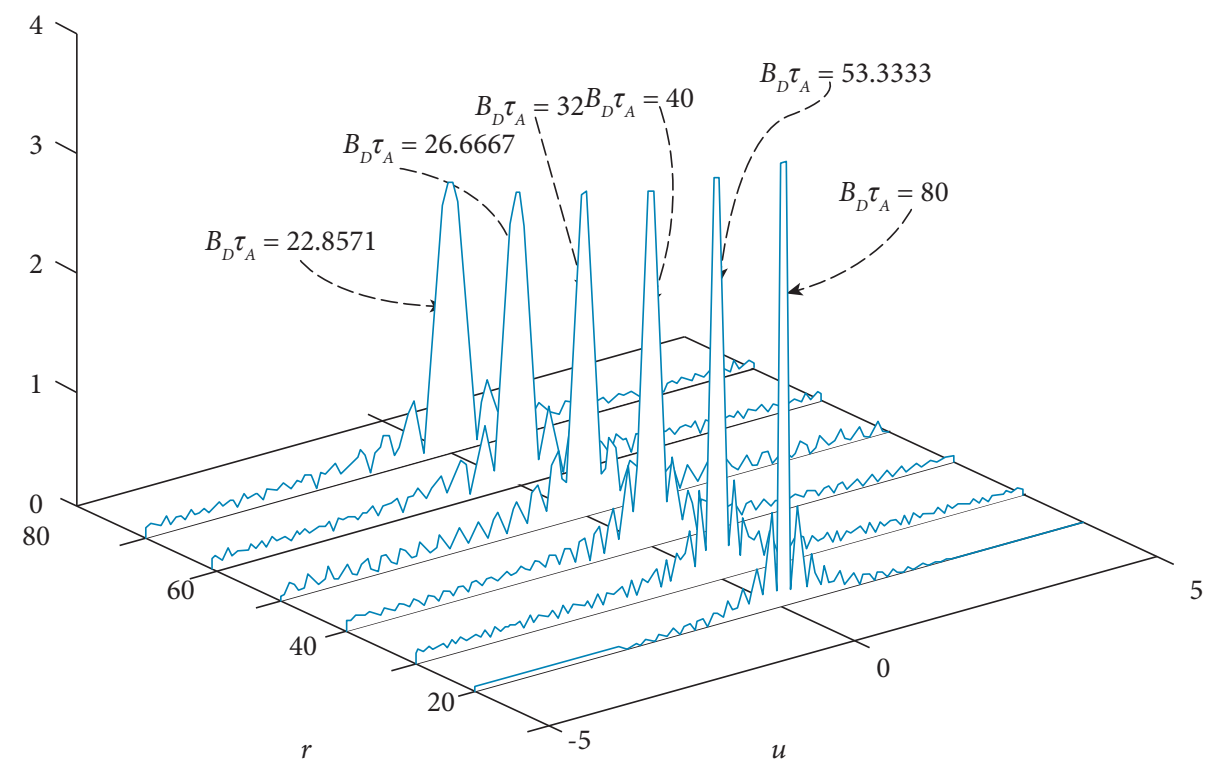

Figure 10. Sinc-like functions resulting from azimuth-matched filtering for targets located at VNR.

\section{EFFECTS OF $\frac{1}{r^{2}}$ FOR THE VNR}

For a given probing region of dimension $10 \mathrm{~m} \times 100 \mathrm{~m}$, and for a radar trajectory located $1 \mathrm{~m}$ away from and parallel to one of its shortest border, the range support extends from $1 \mathrm{~m}$ to $100 \mathrm{~m}$. Such a geometry implies a free space loss dynamic range of about $40 \mathrm{~dB}$. Therefore, a great impact in the relative resolution capability of the radar is expected for targets positioned at the opposite ends of the probing polygon. Two targets, for example, with equal radar cross-section, located at $1 \mathrm{~m}$ and $100 \mathrm{~m}$, respectively, will be registered by the radar with $40 \mathrm{~dB}$ of reflectance difference.

To overcome the impact of free space loss dynamic range in the relative resolution capability for VNR working radars, a small probing sub-area of interest could be defined in such a way that it doesn't allow a difference greater than $3 \mathrm{~dB}$ for the most distant targets inside it. If the shortest range of this small sub-area is taken as $25 \mathrm{~m}$, for example, the largest range must be $35 \mathrm{~m}$, making the useful range swath of such probing area to be only $10 \mathrm{~m}$ long.

SAR operated from medium to large ranges do not exhibit this kind of limitation. For a length swath of, for instance, $100 \mathrm{~m}$, and a radar trajectory $195 \mathrm{~km}$ away from the center of the region to be imaged, the free space loss dynamic range is $0.22 \mathrm{~dB}$. So, in this case, targets with equal radar cross-section, located at the opposite ends, will be registered by the radar with a reflectance difference of only $0.22 \mathrm{~dB}$.

\section{ת-K A-BYU VERSUS MRA-MIT}

Even though some other strategies could be attempted for focusing the image (for instance, the Range-Doppler algorithm), in this project it has been decided to apply the $\omega-\kappa$ algorithm for compressing our raw data. The $\omega-\kappa$ algorithm (Cafforio et al. 1991 ) is well suited for wide apertures and short range swaths (Cumming et al. 2003).

A very good description of the $\omega-\kappa$ algorithm is given in the seminal paper of Cafforio et al. (1991), based on a pulsed radar on which the raw data collected is entirely in the time domain. While this paper does not intend to go any further in describing 
the $\omega-\kappa$ algorithm (the reader could refer to Cafforio et al. 1991, Carrara et al. 1995), it must be pointed out that, when dealing with CW-LFM dechirped raw data, the resulting analytic signal $s_{\mathrm{A}}(t)$ could be considered (Duersch 2004, Tolman 2008, Edwards 2009) in the time domain as (for a point scatterer):

$$
S_{A}(t)=w\left(\frac{t}{\tau}\right) e^{-j 2 \pi f_{\Delta} t} e^{-j 2 \pi f_{0} \Delta(u)}
$$

where $f_{\Delta}=K \Delta$ is, as mentioned before, the target beating frequency.

This approach has been used extensively at BYU (Duersch 2004, Tolman 2008, Edwards 2009). But, also, the resulting analytic signal $s_{\mathrm{A}}(t)$ could be considered in the frequency domain (Charvat 2014):

$$
s_{A}(t)=w\left(\frac{t}{\tau}\right) e^{-j \omega(t) \Delta(u)}
$$

where $\omega(t)=2 \kappa\left(f_{0}+K t\right)$ is the instantaneous angular frequency of the echo.

This approach has been used by Charvat (2014) at MIT while calling the $\omega-\kappa$ algorithm by the equivalent name of Range Migration Algorithm (RMA). The $\omega-\kappa$ algorithm (BYU) follows the sequence:

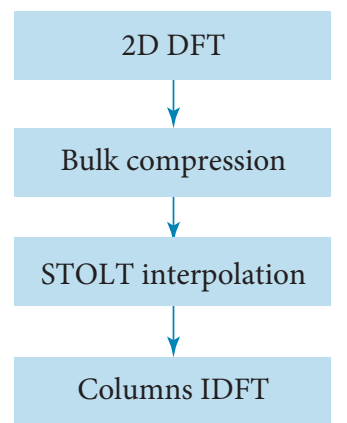

This approach will be abbreviated as $\omega-\kappa \mathrm{A}-\mathrm{BYU}$.

On the other hand, the RMA (MIT) follows the sequence:

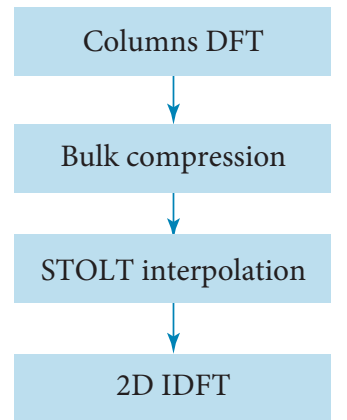

and will be abbreviated here as RMA-MIT.

Both said approaches have been tested over simulated and real raw data with very similar results. In the next section, the results for the RMA-MIT will be presented. 


\section{PROBING CAMPAIGN AND RESULTS}

Several probing experimental campaigns were completed with a rectangular area of $12 \mathrm{~m} \times 100 \mathrm{~m}$, configured as a testing polygon. Two pairs of aluminum corner reflectors of $71 \mathrm{~cm}$ and $86 \mathrm{~cm}$ of height, respectively, were constructed. Table 1 shows the estimated RCS for these reflectors.

Table 1. Corner reflector height $(L)$ and its radar cross-section (RCS).

\begin{tabular}{c|c} 
Height $L$ & Theoretical RCS $\left(\sigma=\frac{4 \pi}{3} \frac{L^{4}}{\lambda_{0}^{2}}\right)$ \\
\hline $71 \mathrm{~cm}$ & $70 \mathrm{~m}^{2}$ \\
$86 \mathrm{~cm}$ & $150 \mathrm{~m}^{2}$
\end{tabular}

The four corner reflectors were arranged geometrically into the testing polygon as shown in Fig. 11 and Fig. 12.

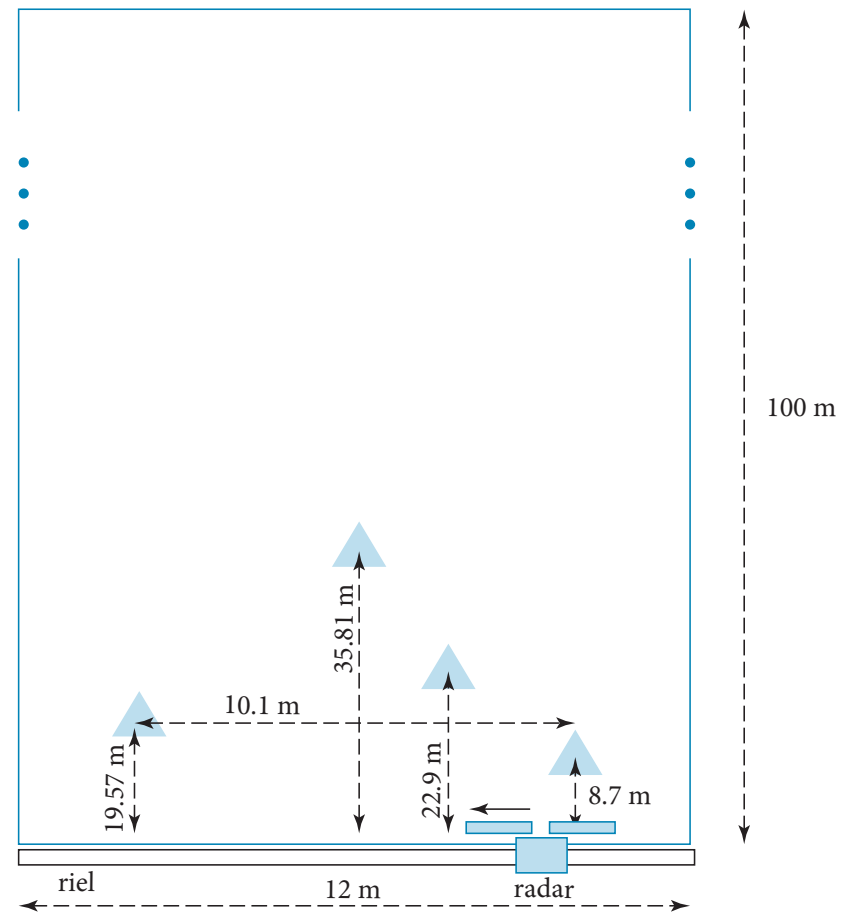

Figure 11. Corner reflectors geometrical arrangement into the testing polygon.

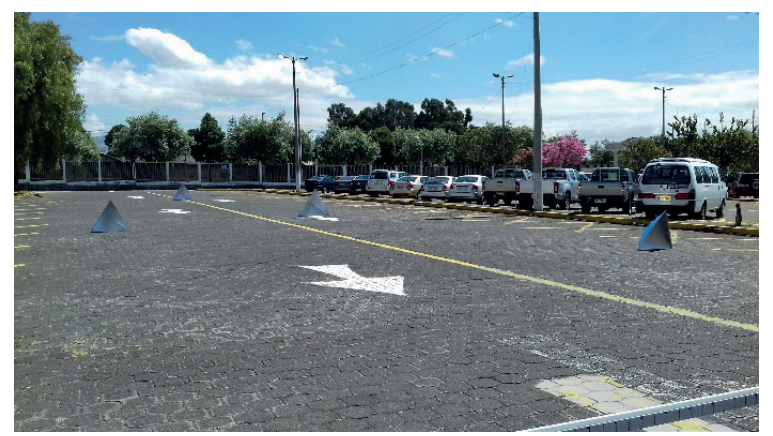

Figure 12. Probing polygon snapshot. 
Likewise, the CW-LFM radar was attached to a little cart and mounted over a bar $12 \mathrm{~m}$ long positioned $1.3 \mathrm{~m}$ over the ground. This bar was laid out along one of the shortest sides of the polygon and marked with little vertical lines every $10 \mathrm{~cm}$. Then, the radar was manually moved over the track while pointing perpendicularly to the polygon following a stop-and-go pattern. In Fig. 12 and Fig. 13, snapshots of the probing polygon and the capturing procedure are shown, respectively.

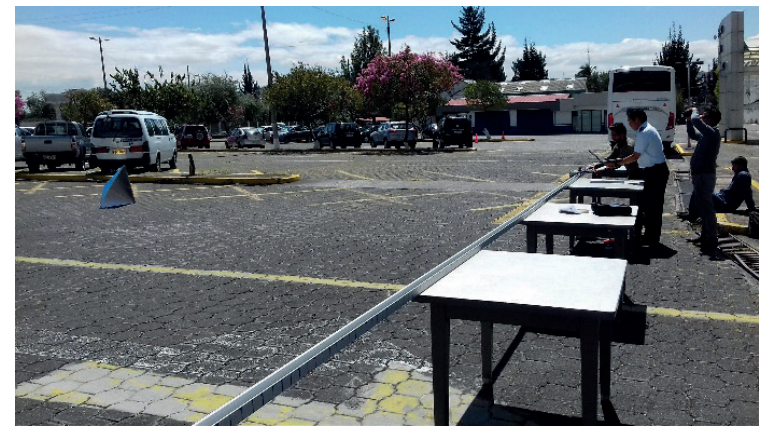

Figure 13. Capturing procedure snapshot.

The data was collected using Audacity ${ }^{\circledR}$, and, after removing irrelevant data with GNU Octave, a $125 \times 226$ matrix of raw data was constructed. Afterwards, said data was processed using the RMA-MIT. Figures 14 and 15 show two focused images.

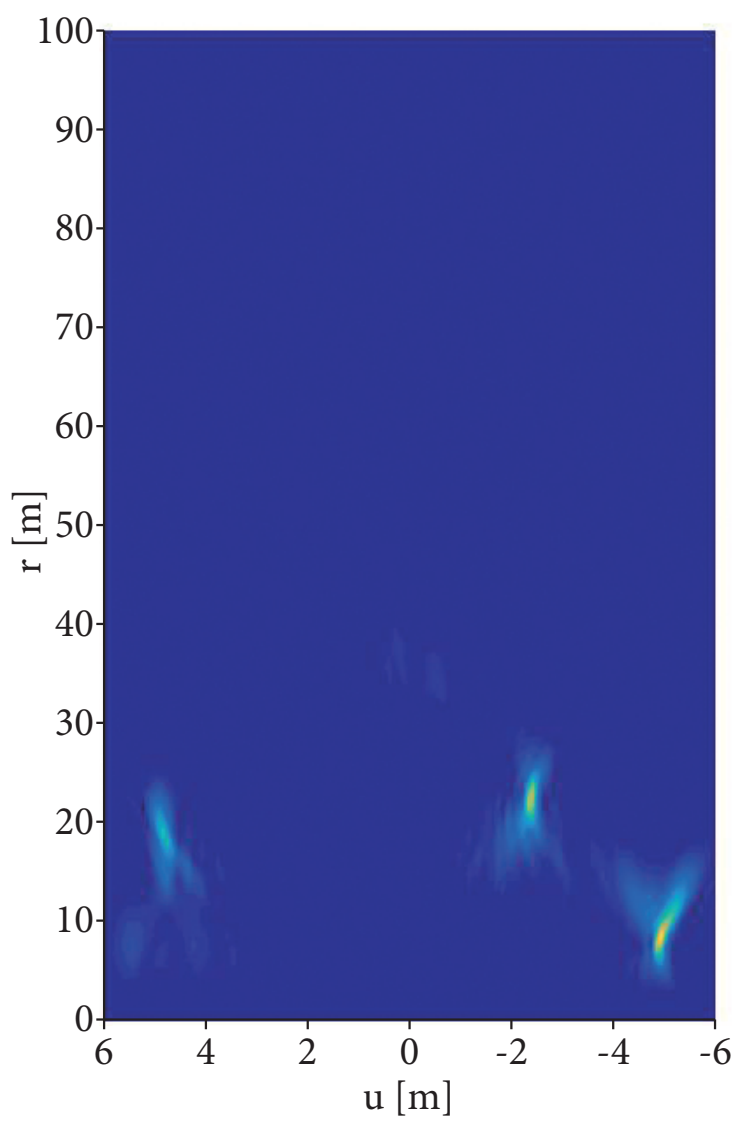

Figure 14. Focused image from raw data using the RMA-MIT in the range from 0 to 100 meters. 


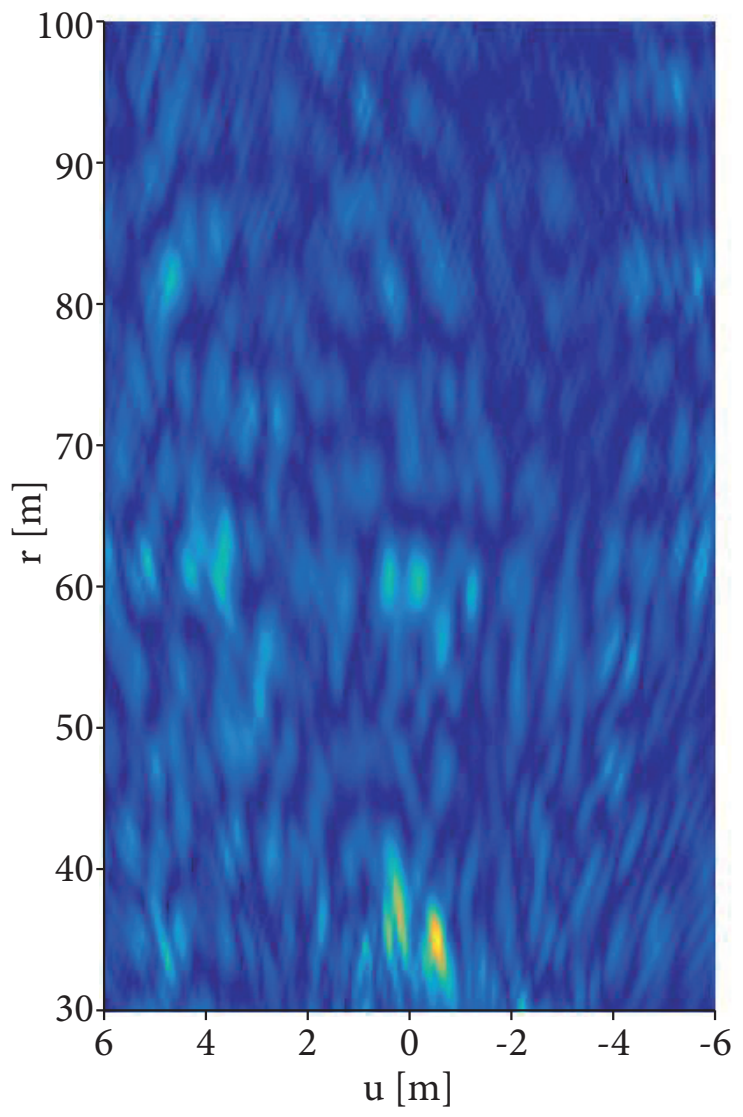

Figure 15. Focused image from raw data using the RMA-MIT in the range from 30 to 100 meters.

In the image displayed by Fig. 14, the farther target is not visible due to the $1 / r^{2}$ effect and its poorer cross-range phase history. In Figure 15 , it can be seen that the most distant target has emerged after the more reflective and easier to focus competing targets were not included.

Finally, to evaluate qualitatively the performance of the radar, the point spread function (PSF) for the nearest target obtained via simulation and as measured by the radar were plotted, and are presented in Figs. 16 and 17, respectively. The simulated PSF was retrieved using a mathematical simulator of the entire system. The actual PSF of the target was obtained from the radar and the RMA-MIT procedure. The similarity between them can be seen in the aforementioned figures.

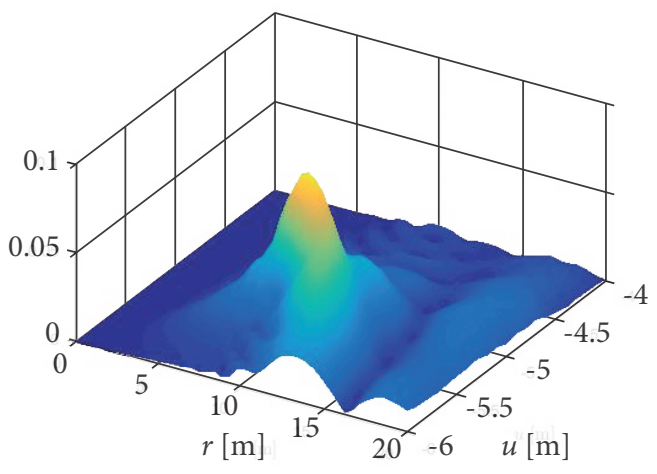

Figure 16. Nearest target PSF obtained via simulation. 


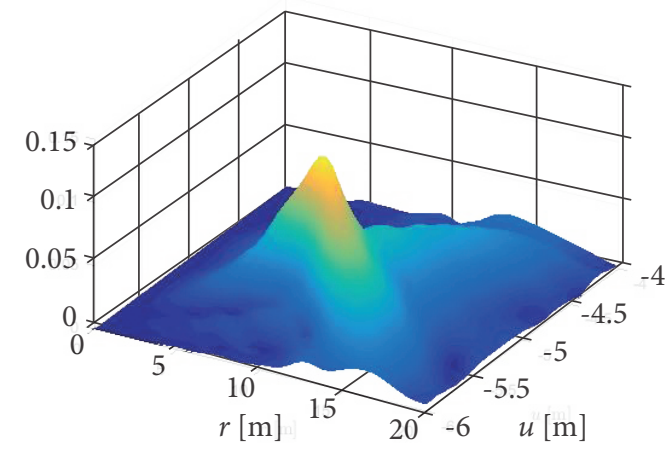

Figure 17. Nearest target actual PSF.

\section{CONCLUSION}

The LFM-CW MIT radar was implemented at the Ecuadorian Space Institute for the purpose of gaining an initial expertise in SAR field. The implemented radar was tested in a small probing polygon for which near and very near ranges were defined in order to estimate the focusing capabilities. The geometrical configuration of the test was theoretically analyzed, and some preliminary considerations about the focusing strategies were established. Finally, an imaging campaign was carried out, obtaining results that agree well with the theory.

\section{AUTHOR'S CONTRIBUTION}

Conceptualization, Zozaya A; Data Curation, Zozaya A; Formal Analysis, Zozaya A; Funding Acquisition, Bolaños R and Zozaya A; Investigation, Zozaya A; Methodology, Zozaya A; Project Administration, Zozaya A and Bolaños R; Resources, Zozaya A and Bolaños R; Software, Zozaya A; Supervision, Zozaya A; Validation, Zozaya A; Visualization, Zozaya A; Writing - Original Draft, Zozaya A; Writing - Review \& Editing Zozaya A and Bolaños R.

\section{REFERENCES}

Cafforio C, Prati C, Rocca F (1991) SAR Data Focusing Using Seismic Migration Techniques. IEEE T Aero Elec Sys 27:194-207. https: // doi.org/10.1109/7.78293

Carrara WG, Goodman RS, Majewski RM (1995) Spotlight Synthetic Aperture Radar: Signal Processing Algorithms. Boston/London: Artech House.

Charvat GL (2011a) Doppler Experiment using the MIT IAP 2011 Laptop Based Radar. Build a Small Radar System Capable of Sensing Range, Doppler, and Synthetic Aperture Radar Imaging.

Charvat GL (2011b) MIT IAP 2011 Laptop Based Radar: Block Diagram, Schematics, Bill of Material, and Fabrication Instructions. Build a Small Radar System Capable of Sensing Range, Doppler, and Synthetic Aperture Radar Imaging

Charvat GL (2011c) Ranging Experiment Using the Mit lap 2011 Laptop Based Radar. Build a Small Radar System Capable of Sensing Range, Doppler, and Synthetic Aperture Radar Imaging. 
Charvat GL (2011d) Student Field Experiment Results using the MIT IAP 2011 Laptop Based Radar. Build a Small Radar System Capable of Sensing Range, Doppler, and Synthetic Aperture Radar Imaging.

Charvat GL (2011e) Synthetic Aperture Radar (SAR) Imaging using the MIT IAP 2011 Laptop Based Radar. Build a Small Radar System Capable of Sensing Range, Doppler, and Synthetic Aperture Radar Imaging.

Charvat GL (2011f) Synthetic Aperture Radar (SAR) Experiment using the MIT IAP 2011 Laptop Based Radar. Build a small radar system capable of sensing range, doppler, and synthetic aperture radar imaging.

Charvat GL (2014) Small and Short-Range Radar Systems. Boca Raton: CRC Press.

Cumming IG, Neo YL, Wong FH (2003) Interpretations of the Omega-K Algorithm and Comparisons with other Algorithms. Geoscience and Remote Sensing Symposium. IGARSS 'O3. Proceedings. IEEE International. https://doi.org/10.1109/IGARSS.2003.1294142

Duersch MI (2004) BYU micro-sar: a very small, low-power LFM-CW synthetic aperture radar (MS thesis). Provo/UT: Brigham Young University.

Edwards MC (2009) Design of a continuous-wave synthetic aperture radar system with analog dechirp (MS thesis). Provo/UT: Brigham Young University.

Fenn AJ (2011) Antenna Design for the Laptop Radar Project. Build a Small Radar System Capable of Sensing Range, Doppler, and Synthetic Aperture Radar Imaging.

Giraldez AE (2004) SAOCOM-1 Argentina L-Band SAR Mission Overview. ESA Special Publication.

Moreira A, Prats-Iraola P, Younis M, KrieGer G, HajnseK I, Papathanassiou KP (2013) A Tutorial on Synthetic Aperture Radar. IEEE GEOSC REM SEN M https://doi.org/10.1109/MGRS.2013.2248301

Tolman MA (2008) A detailed look at the omega-k algorithm for processing synthetic aperture radar data (MS thesis). Provo/UT: Brigham Young University.

Williams JH (2011) Modular System RF Design. Build a Small Radar System Capable of Sensing Range, Doppler, and Synthetic Aperture Radar Imaging.

Zaugg EC (2010) Generalized Image Formation for Pulsed and LFM-CW Synthetic Aperture Radar (PhD thesis). Provo/UT: Brigham Young University. 